\title{
Challenges and opportunities for the use of biomarkers of rare genetic diseases
}

\begin{abstract}
Rare genetic diseases are a general classification of some 6000 disorders impacting millions of people. Most rare genetic disorders are life threatening or have a serious impact on the quality of life, often requiring lifelong medical intervention and support. Despite the large number of rare genetic diseases, relatively few drugs have been approved (approximately 400). The challenges facing developers of treatments for rare diseases are multiple. Surrogate biomarkers for rare diseases hold great promise to enable successful drug development, long term management of disease and patient empowerment. Rare disease drug development is often challenged by a lack of scientific knowledge about the disease, small patient populations, expensive trials, regulatory hurdles, and high cost of commercial failure. However, great opportunities exist for the treatment of rare diseases. Large and small biopharmaceutical companies have proven that developing and marketing treatments for rare genetic diseases is viable. Bio analytical tests have been developed for many disorders; new technologies for hand-held, self-administered tests are within reach. Monitoring devices and healthcare networking applications are ideal for enabling successful treatments for people suffering from rare genetic diseases.
\end{abstract}

Volume I Issue 2 - 2014

\section{Erik D Foehr}

Senior Partner, Strategic Partners Consulting, USA

Correspondence: Erik D Foehr, Senior Partner, Strategic Partners Consulting, 1729 King Street, Suite 100,Alexandria,VA 223 |4, USA, Tel 4I53204689, Email Erik.Foehr@sp2lc.com

Received: December 06, 2014 | Published: December 18, 2014

Keywords: rare genetic disease, biomarker, self-monitoring, clinical trial

Abbreviations: ERT, enzyme replacement therapy; GAG, glycosaminoglycan; PAH, phenylalanine hydroxylase; Phe, phenylalanine; PKU, phenylketonuria; MPS, mucopolysaccharidosis; ODDT, open drug discovery teams; FDA, food and drug administration

\section{Introduction}

The 1983 US Orphan Drug Act resulted in a significant and sustained increase in treatments for rare diseases. The thousands of rare genetic diseases affect over 10million Americans. A rare disease is defined as a disorder with a prevalence of less than 200,000 in the United States. ${ }^{1}$ Thousands of drugs are being developed for rare diseases; however, the majority of treatments are for rare oncology and infectious diseases. A few but noteworthy and successful treatments for rare genetic diseases will be discussed. Enzyme replacement therapy (ERT) for Mucopolysaccharidoses has improved the quality of life for people with these rare genetic diseases. ${ }^{2}$ Another rare genetic disease, Phenylketonuria (PKU), has benefited from new treatment strategies. ${ }^{3}$ The economic impact of these new rare disease treatments to drug developers, patients, insurers/governments is significant. The successful development of treatments for rare genetic diseases requires a systematic and coordinated approach to product development. The study of rare diseases is challenging - but the solution may require a "communal approach", one that builds networks to allow broader access to trials. Since the Orphan Drug Act, over 400 treatments have been approved. Large and small pharmaceutical companies have benefited. Several new orphan drugs are priced over $\$ 150,000$ per patient per year. ${ }^{4}$ These successes are due to many factors.

Biomarkers/surrogate endpoints help reduce clinical trial size by enabling flexible study designs (i.e. adaptive design). This is because continuous measures of a biomarker impart greater statistical power in analysis than the traditional outcome or endpoint measures. ${ }^{5}$ The ability to share information about the rare genetic disease etiology and natural history provides drug developers with an advantage. New bio analytical technologies can enable point of care and self monitoring for rare diseases, but validation of biomarkers and the analytical measurement is difficult. Validation of biomarkers may require decades of research and clinical experience with rare genetic diseases. The disease pathophysiology must be well understood. A clinically relevant and valid biomarker needs to have a direct relationship to the disease state and provide predictive value. For instance the MPS disorders have an abnormal accumulation of glycosaminoglycans (GAGs) in cells and tissues. Many clinical studies have demonstrated a correlation with reduced GAG in urine and blood with better clinical outcomes and quality of life measures. ${ }^{2}$ Similarly people with PKU, an autosomal recessive disorder caused by mutation in the phenylalanine hydroxylase (PAH) gene, have elevated phenylalanine (PHE). Accumulation of Phe causes progressive irreversible neurological impairment. The severity of the phenotype directly correlates with blood Phe levels. Drugs have been approved for both MPS and PKU disorders with strong biochemical data supporting the drug development process. Therefore, the identification, measurement and monitoring of biochemical markers pose challenges and opportunities for the successful treatment of rare genetic diseases.

\section{Discussion}

Due to the lack of measurable and recognized clinical endpoints for rare genetic diseases, the use of biomarkers as surrogates has become vitally important. Technologies exist to enable successful drug development and treatment. Finding patients with rare diseases to enroll, study and treat has not been easy. Clinical trials for rare genetic diseases often need to open sites in multiple countries just to cobble together a few dozen patients for the clinical trials. ${ }^{6}$ Social media platforms can be useful for sharing information about the disease. Sharing data about the natural history of a rare disease would benefit patients and drug developers. Health information technology companies such as Athena Health are organizing data to facilitate more efficient use of healthcare resources. The Epocrates App is 
used by patients and providers to improve patient engagement. This could help build a community to support rare genetic disease research and treatments. For instance Ekins et al., ${ }^{7}$ developed the Open Drug Discovery Teams (ODDT) App that provides a magazine style interface to gather and present information about rare and neglected diseases, such as Sanfilippo syndrome. Sanfilippo Syndrome is one of 11 inherited genetic disease of the MPS family of disorders that result in failure to breakdown GAG. The accumulation of GAGs in cells and tissues result in a number of disabilities, such as reduced mobility, sleep apnea, and organ function problems. Clinical trials that study MPS disorders often measure walk distance and collect information on sleep and other biometric information. Several innovative companies are developing tools to gather biometric information that could be employed for rare disease studies. For instance the EarlySense piezoelectric sensor provides contactless respiratory and heart rate monitoring through monitors located under the mattress. ${ }^{8}$ The FDA recently approved wrist worn devices that are intended for the acquisition and analysis of physical activity during daily living and sleep. ${ }^{9}$ The BodyMedia FIT, MotionWatch 8 and PRO-Diary may be useful to gather information from MPS patients that could be shared with the patient community, researchers, regulators, and drug developers to better design, study and treat people with rare genetic disorders such as the MPS diseases.

Biochemical markers of disease also present great opportunity Advancements in genomics and biomedical research have allowed researchers to identify the genetic etiology of over 4500 diseases, compared to only 50 diseases 20years ago. ${ }^{10}$ However, rare genetic diseases may still require decades of study to identify useful biomarkers. A validated biomarker should be predictive of the disease state. The biomarker should increase or decrease in a statistically significant way with disease severity or treatment. Reaching statistical significance can be difficult with a small heterogeneous population, often found in rare genetic diseases. Often multiple biomarkers such as a panel of metabolites or cytokines can be utilized. A multiplexed approach may be informative, but the data can be complex and expensive to interpret and gather. Biomarkers are typically measured from blood (plasma or serum), urine, cerebral spinal fluid, or other matrix. However, collecting enough quality samples can be challenging, especially in pediatric or neonatal populations. ${ }^{11}$ For example, the level of GAGs in urine is elevated in the MPS diseases and can be reduced following treatment with ERT. Could new technologies be applied to this family of rare diseases? GAGs can be measured by many analytical techniques, including mass spectrometry, immunoassay, and colorimetric analysis. ${ }^{12}$ Classically GAGs were measured in urine using spectrophotometric analysis of the dimethylmethylene blue dye bound to sulfate groups on the GAG polymer. Newer techniques measure the specific disaccharides that comprise GAGs and provide biochemical information about the disease state. However, these bioanalytical techniques require special sample processing and large expensive instruments. The challenge going forward is to adapt the detailed biochemical and analytical knowledge to cheaper, faster tests. Eventually researchers and patients may have the ability to self monitor with hand held devices. With a concerted effort it may be possible to develop a self administered test to measure urinary GAG levels, much like a pregnancy test.

PKU can be treated with a specialized Phe-free diet and in some cases a drug called Tetrahydrobiopterin (aka Kuvan), that acts as a molecular chaperone for the defective PAH enzyme. The treatments reduce the levels of Phe and have demonstrated improved clinical outcomes. Monitoring Phe levels is critical to disease management.
The Phe test is typically performed on blood samples collected at home and then sent to a central testing lab for analysis using liquid chromatography-mass spectrometry. This test takes a couple weeks to perform and the result is sent to the patient's physician. Weeks may pass before a patient is aware of any changes to their disease status. A medical App, called myPKU is being used by patients and family members to help manage Phenylketonuria by tracking diet and blood test results (Nutricia Ltd). In a randomized clinical trial of PKU patients, Hoedt et al. ${ }^{13}$ demonstrated increased self-management of the disease by providing direct access to blood Phe. Direct to patient access to the biomarker data was safe and a preferred method to obtain the information. A hand-held, real-time testing device for PKU may be on the horizon. At the 2014 National PKU Alliance Conference, Dr. Tom Franklin outlined efforts to measure Phenylalanine in blood from a finger stick (much like a glucose meter) down to $1 \mathrm{mg} / \mathrm{dL}$ with the appropriate specificity and accuracy. The Phe monitor may work by immobilizing an enzyme such as Phenylalanine dehydrogenase onto a polymer test strip. ${ }^{14}$ The blood would be drawn into the test strip and Phe measured via an electrochemical reaction. This approach has been used for other hand held monitors and could be commercially viable considering the decades of experience with glucose monitors. Due to the cost of development and small market size, public-private partnerships may be necessary to make hand-held monitoring devices available to people with rare genetic diseases. These are the challenges and opportunities of using biomarkers to treat rare genetic diseases.

\section{Conclusion}

Development of robust methods to measure surrogate biomarkers is key to the successful treatment of rare genetic diseases. Due to the small and heterogeneous population of people suffering from rare genetic diseases, new technologies will be needed to measure, collect and analyze data. Test methods to measure biochemical markers of the disease state or response to treatment can be modernized to enable self monitoring. For example rare genetic disease biomarkers could be measured by using testing platforms similar to glucose meters or urine pregnancy tests. With appropriate privacy controls, the data collected could be shared with researchers, clinicians, or fellow patients through social media or disease specific "Apps" from smart phones. This information would help doctors design and manage better clinical trials with endpoints appropriate for the rare disease, and help empower individuals. The Phe monitor for PKU disease management, or motion tracker devices for establishing clinical endpoints are examples of how new technologies are enabling the treatment of rare genetic diseases. Collaboration between patient advocacy groups, regulators, physicians, device manufacturers and biopharmaceutical companies will be required to achieve the goal of treating rare genetic diseases.

\section{Acknowledgements}

None.

\section{Conflict of interest}

Author declares that there is no conflict of interest.

\section{References}

1. Policies and Guidelines. International Rare Disease Research Consortium (IRDiRC). 2013:1-12.

2. Martell L, Lau K, Mei M, et al. Biomarker analysis of Morquio syndrome: Identification of disease state and drug responsive markers. Orphanet J Rare Dis. 2011;6:84. 
3. Strisciuglio P, Concolino D. New strategies for the treatment of Phenylketonuria (PKU). Metabolites. 2014;4(4):1007-1017.

4. Stephens J, BlazynkiC. Rare disease landscape: will the blockbuster model be replaced? Expert Opinion on Orphan Drugs. 2014;2(8):797-806.

5. Gagne JJ, Thompson L, Keefe KO, et al. Innovative research methods for studying treatments for rare diseases: methodological review. $B M J$. 2014;349:g6802.

6. Shurin S, Krishner J, Groft SC. Clinical trials in BMT: ensuring that rare diseases and rarer therapies are well done. Biol Blood Marrow Transplant. 2012;18(suppl1):8-11.

7. Ekins S, Clark AM, Williams AJ. Open drug discovery teams: A chemistry mobile App for collaboration. Mol Inform. 2012;31(8):585-597.

8. Ben-Ari J, Zimlichman E, Adi N, et al. Contactless respiratory and heart rate monitoring: Validation of an innovative tool. J Med Eng Technol. 2010;34(7-8):393-398.

9. Comstock J. FDA clears two wrist worn fitness trackers for clinical trials. Mobihealthnews; 2014
10. Cure The Process. Accelerating Biotech Innovation for Rare Disease Treatments. 2014.

11. Bai JP, Barrett JS, Burckart GJ, et al. Strategic Biomarkers for drug development in treating rare diseases and diseases in neonates and infants. AAPS J. 2013;15(2):447-454.

12. Tomatsu S, Shimada T, Mason RW, et al. Establishment of glycosaminoglycan assays for mucopolysaccharidoses. Metabolites. 2014;4(3):655-679.

13. Ten Hoedt AE, Hollak C, Boelen C, et al. My PKU: Increasing selfmanagement in patients with Phenylketonuria. A randomized controlled trial. Orphanet J Rare Dis. 2011;6:48.

14. Omidinia E, Khanehzar A, Nasrin S, et al. Covalent immobilization of phenylalanine Dehydrogenase on Glutaraldehyde modified poly (3,4-ethylenedioxy) Thiohene: Poly(styrenesulfonate)/polyvinyl alcohol conducting polymer composite films for electrochemical detection of L-phenylalanine. Anal Bioanal Electrochem. 2013;5(5):597-608. 